\title{
Controlled active exercise after open reduction and internal fixation of hand fractures
}

\author{
Dongkeun Jun, Jaehyun Bae, Donghyeok Shin, Hyungon Choi, Jeenam Kim, Myungchul Lee \\ Department of Plastic and Reconstructive Surgery, Konkuk University School of Medicine, Seoul, Korea
}

\begin{abstract}
Background Hand fractures can be treated using various operative or nonoperative methods. When an operative technique utilizing fixation is performed, early postoperative mobilization has been advocated. We implemented a protocol involving controlled active exercise in the early postoperative period and analyzed the outcomes.

Methods Patients who were diagnosed with proximal phalangeal or metacarpal fractures of the second to fifth digits were included $(n=37)$. Minimally invasive open reduction and internal fixation procedures were performed. At 3 weeks postoperatively, controlled active exercise was initiated, with stress applied against the direction of axial loading. The exercise involved pain-free active traction in three positions (supination, neutral, and pronation) between 3 and 5 weeks postoperatively. Postoperative radiographs and range of motion (ROM) in the interphalangeal and metacarpophalangeal joints were analyzed.

Results Significant improvements in ROM were found between 6 and 12 weeks for both proximal phalangeal and metacarpal fractures $(P<0.05)$. At 12 weeks, 26 patients achieved a total ROM of more than $230^{\circ}$ in the affected finger. Postoperative radiographic images demonstrated union of the affected proximal phalangeal and metacarpal bones at a 20-week postoperative follow-up.

Conclusions Minimally invasive open reduction and internal fixation minimized periosteal and peritendinous dissection in hand fractures. Controlled active exercise utilizing pain-free active traction in three different positions resulted in early functional exercise with an acceptable ROM.
\end{abstract}

Keywords Hand / Fracture / Open reduction / Exercise

\author{
Correspondence: Myungchul Lee \\ Department of Plastic and \\ Reconstructive Surgery, Konkuk \\ University School of Medicine, 120-1 \\ Neungdong-ro, Gwangjin-gu, Seoul \\ 05030, Korea \\ Tel: +82-2-2030-7639 \\ Fax: +82-2-2030-7733 \\ E-mail:mcle1999@gmail.com
}

This article was presented at the 10th Research and Reconstruction Forum on July 25, 2020.

Received: August 31, 2020 • Revised: November 8, 2020 • Accepted: November 13, 2020

pISSN: 2234-6163 • elSSN: 2234-6171 • https://doi.org/10.5999/aps.2020.01739• Arch Plast Surg 2021;48:98-106

\section{INTRODUCTION}

Phalangeal fractures are the most common fractures of the hand, followed by metacarpal fractures [1]. Both non-surgical and surgical treatment methods exist for these two types of fractures; however, non-surgical methods have limitations, such as long-term immobilization and difficulty in maintaining rota- tional stability [2]. Previous anatomical studies have reported that deformities of the phalangeal or metacarpal bones can lead to the development of an extensor lag and pseudoclawing [3]. Therefore, it is important to accurately reduce these fractures and to maintain reduction.

Although there is some variation in the indications for surgical reduction of phalangeal fractures in the literature, surgical inter- 
ventions are recommended for periarticular fractures with lateral instability, displaced phalangeal neck fractures, displaced proximal phalangeal base fractures, and diaphyseal fractures with articular involvement [4].

Surgical treatment for metacarpal fractures may be appropriate if a rotational deformity or angulation was not properly corrected, or if correction was not maintained, after a non-surgical intervention. Although the prognosis of angulation varies somewhat depending which digit is injured, slight residual angulation after reduction can be monitored after splint application [5].

Various closed and open surgical techniques have been proposed for the treatment of phalangeal fractures and metacarpal fractures, including closed reduction and percutaneous pinning, intramedullary nailing, open reduction and screw fixation, and plate fixation [5-8].

Correcting fracture angulation by performing and maintaining accurate reduction is a crucial aspect of treating phalangeal and metacarpal fractures $[5,6]$. Of the aforementioned surgical techniques, biomechanically stable methods should be selected; however, objectively comparing the reported methods is difficult due to differences among studies in strength measurement methods, fixation device characteristics, and fracture patterns. In addition, since excessive periosteal and peritendinous dissection may restrict the postoperative range of motion of the joint even after stable fixation, it is important to strike a balance between stable fixation and minimal dissection [4]. Although the extent of dissection may be reduced by a minimal incision and internal fixation, careful observation is required to ensure postoperative stability, and appropriate active exercises that do not induce dislocation can be performed to facilitate recovery at 2-4 postoperative weeks after limited stability has been achieved [9].

In this study, we report the radiologic outcomes and range of joint motion after minimally invasive open reduction and internal fixation of proximal phalangeal or metacarpal fractures, followed by controlled active exercise.

\section{METHODS}

\section{Study subjects}

Thirty-seven patients who were diagnosed as having a single proximal phalangeal or metacarpal fracture of the second to fifth digits of the hand and underwent open reduction and internal fixation from March 2015 to December 2019 were enrolled. The patients underwent radiographic imaging preoperatively and postoperatively and were followed up for a retrospective evaluation. The study protocol conformed to the ethical guidelines of the Declaration of Helsinki, and written informed con- sent was obtained from the patients. The study was approved by the institutional review board (IRB No. KUMC 2020-03-013).

Thumb fractures were excluded from the study, as the postoperative immobilization and recovery period and rehabilitation exercises could be significantly different from those for other digits. We also excluded cases where additional operative procedures were required for concomitant tendon or muscle injuries, which were present at the same site as the phalangeal or metacarpal fractures, as such procedures may affect the time to union or the progression of range of motion during postoperative wound recovery. Intra-articular fractures were also excluded, as the prognosis could be significantly different.

A total of 37 patients with a single proximal phalangeal or metacarpal fracture of the second to fifth digits underwent open reduction and internal plate fixation. Patient characteristics, such as age, sex, follow-up period, and fracture site, were analyzed (Table 1). The average age of the patients was 42 years. There were seven women and 30 men. The average follow-up period was 16 months. The fracture site was the proximal phalanx in 12 cases and the metacarpal bone in 25 cases. The plate was removed at an average of 24 weeks (range, 20-31 weeks) after surgery in 16 cases.

\section{Operative technique, postoperative care, and exercise therapy}

During surgery, the fracture site was confirmed by C-arm fluoroscopy, and the corresponding fracture site was marked on the skin and soft tissue using a 25-gauge needle. A Z-shaped incision was made in the direction of the long axis of the proximal phalanx or the metacarpal bone while avoiding the path of the extensor tendon. The incision was made on the ulnar or radial side, considering the fracture line and the proper position for plate fixation. Dissection of the muscles and fascia was performed with care to preserve the main blood vessels and sensory nerves of the dorsum of the hand, and the periosteum around

\section{Table 1. Patient demographics}

\begin{tabular}{lc}
\hline Index & Value \\
\hline No. of patients & 37 \\
Sex & 7 \\
$\quad$ Female & 30 \\
$\quad$ Male & $42 \pm 12(16-65)$ \\
Age (yr) & $16 \pm 5(7-28)$ \\
Observation period (mon) ${ }^{\text {a) }}$ & \\
Fracture site & 12 \\
$\quad$ Proximal phalanx & 25 \\
$\quad$ Metacarpal bone &
\end{tabular}

Values are presented as number or mean \pm SD (range).

a)Time from operation to evaluation. 
the fracture site was elevated. With the fractured digit pulled, a force was applied using a freer or periosteum elevator in the opposite direction of the bone fragment dislocation for reduction. A titanium plate (low-profile 1.2-mm titanium mini plate; Jeil Medical Corp., Seoul, Korea) was fixed to maintain the reduction. After plate fixation, the alignment of the second to fifth digits was confirmed by passive flexion and extension. After confirming the reduction of the proximal phalangeal or metacarpal bone via intraoperative imaging, the periosteum and skin were sutured.

With regard to immobilization, an ulnar or radial gutter splint was maintained for 2 weeks. The splint was applied with the wrist at $30^{\circ}$ extension, the metacarpophalangeal joint at $70^{\circ}$ flexion, and the proximal interphalangeal joint at full extension. The ulnar type was utilized for the ring or small finger, and the radial type for the index or long finger. For additional 1 week, a soft splint was applied from the affected and adjacent fingers to the wrist, using an elastic fabric bandage.

At 3 weeks after surgery, active exercise was initiated, in which a load was applied to the fractured proximal phalangeal or metacarpal bone against the direction of axial loading. Controlled active exercise was performed at 3-5 weeks after surgery using pain-free active traction in three positions of the hand (supination, neutral, and pronation) (Fig. 1). If it was difficult to proceed with the traction exercise or the patient complained of pain during exercise therapy, an easier exercise was used in combination with or instead of the traction exercise. Specifically, a thermoplastic splint was cut to an appropriate size to wrap around the proximal phalanx and was connected by a rubber band to an elastic wrist band, through which a weak load (approximately equal to the tension of the rubber band) was applied for passive flexion and active extension (Fig. 2). Moderate functional activities were allowed starting 4 weeks after surgery.

\section{Measurements and analysis}

Radiographic images (anteroposterior and oblique views) obtained preoperatively, postoperatively, and during follow-up were reviewed to determine whether the fracture site was fused. The range of motion of the interphalangeal and metacarpophalangeal joints was measured at postoperative weeks 6 and 12, and differences between these time points were analyzed. Furthermore, the observed ranges of motion were compared with those reported in previous studies, in which exercise therapy was performed after open reduction and internal fixation of

\section{Fig. 1. Controlled active exercise with pain-free active traction}

Strategic exercises were performed using a Thera-Band in three positions-namely, supination $(A, B)$, neutral $(C, D)$, and pronation $(E, F)$-between 3 and 5 weeks postoperatively. The proximal and distal interphalangeal joints were flexed to hold the elastic band with minimal force. The patient's elbow and shoulder joints were utilized to perform the active traction exercises.
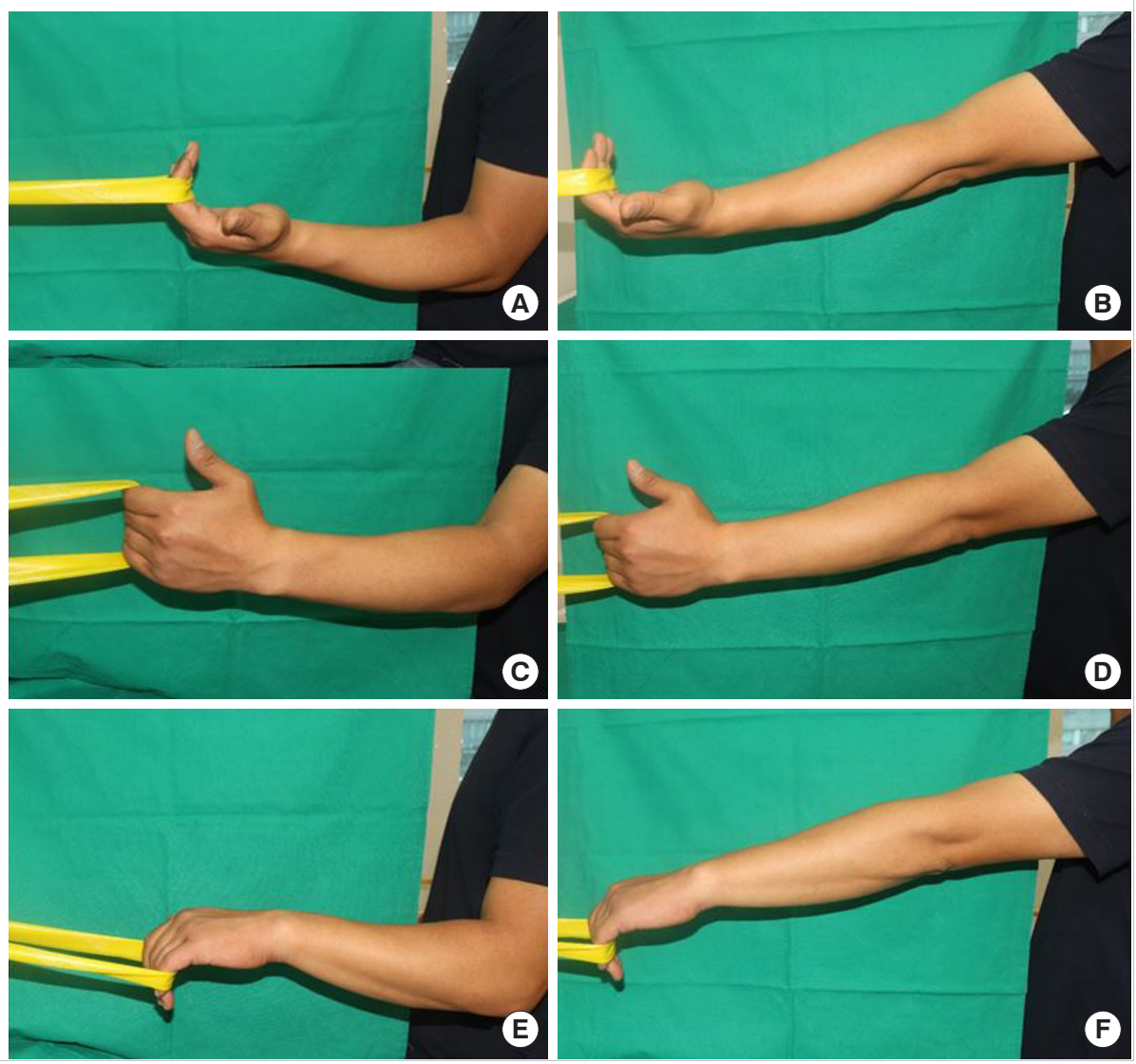
Fig. 2. Minimal loading active exercise with Aquaplast splint

A thermoplastic splint was molded and applied on the proximal phalanx. The link between the splint and rubber band led to gradual flexion of the metacarpophalangeal joint. Active extension $(A)$ and passive flexion (B) were possible.
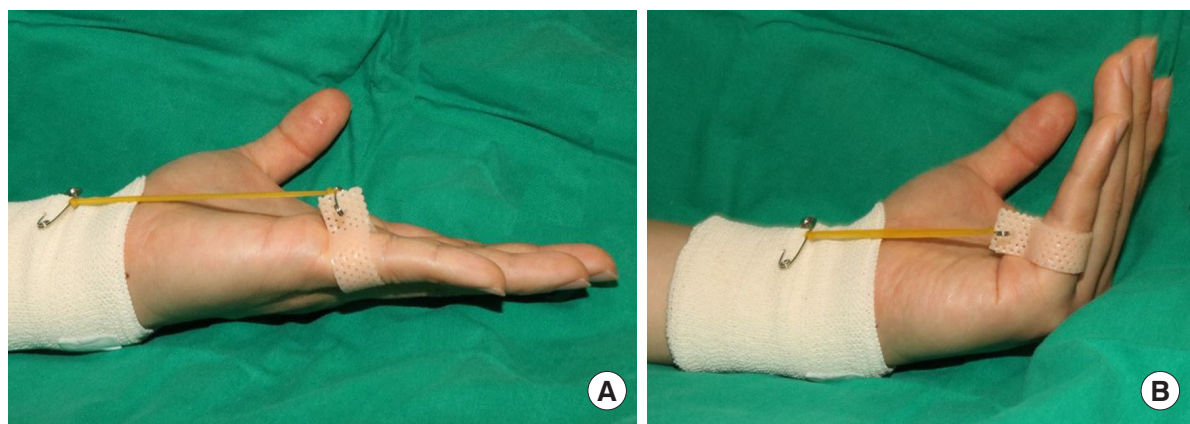

Fig. 3. A case of metacarpal bone fracture

A 30-year-old male patient presented with a fifth metacarpal neck fracture of the right hand (A). Open reduction and internal plate fixation were performed. Bony union had been achieved at a postoperative follow-up of 18 weeks (B). The finger joints had a full range of motion (metacarpophalangeal joint $90^{\circ}$, proximal interphalangeal joint $100^{\circ}$, distal interphalangeal joint $70^{\circ}$ ) without limitation (C, D). The operative wound had a minimal scar (E, F).
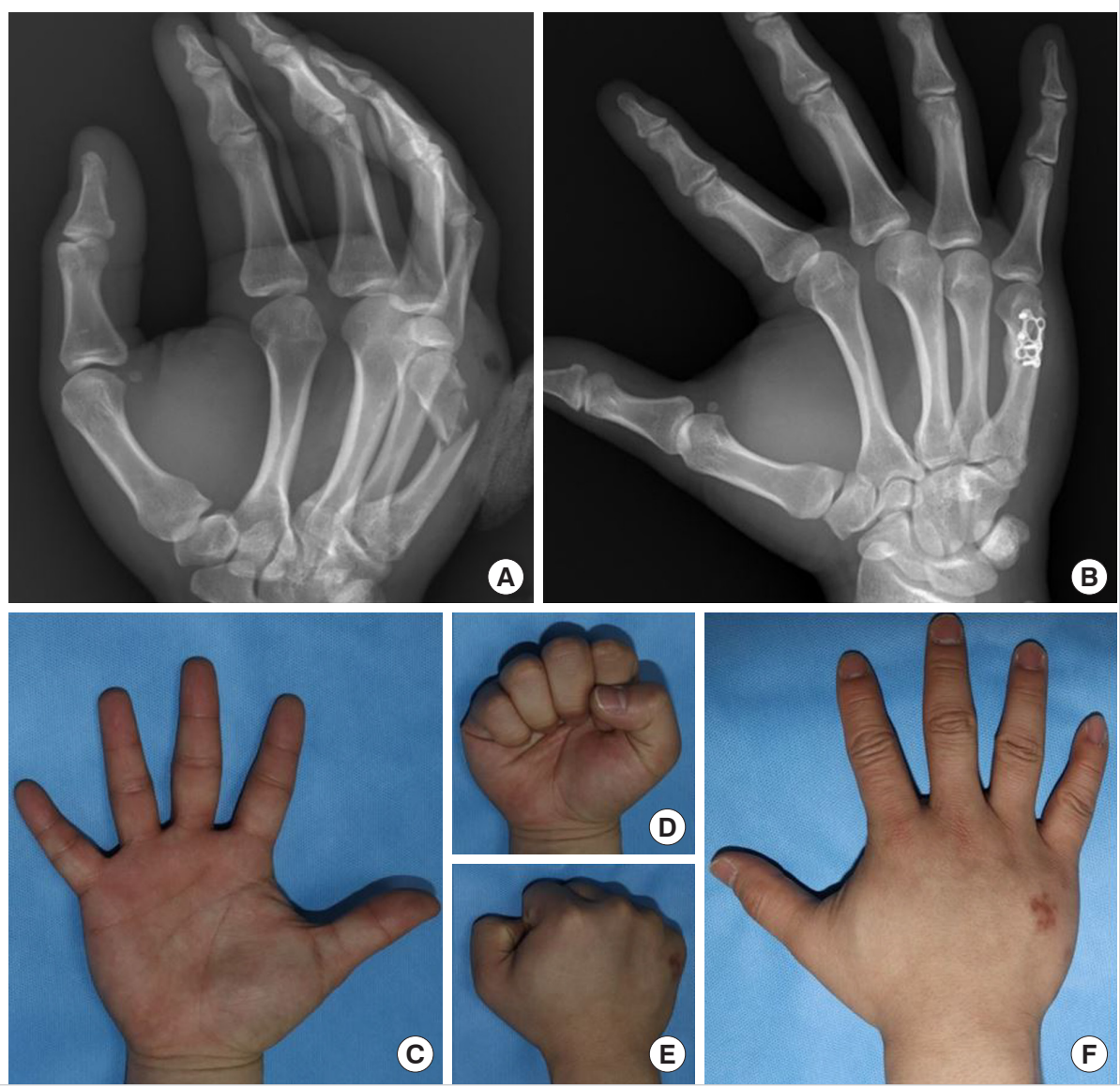

proximal phalangeal or metacarpal fractures $[9,10]$.

For statistical analysis, the paired t-test was used to compare the range of motion before and after surgery within a single group. Intergroup analysis using data on changes in the range of motion was conducted using the unpaired $t$-test. The differences between the range of motion at 6 and 12 weeks were calculated for each joint in the proximal phalangeal and metacarpal bone fracture groups for intergroup comparisons. Standard software (SPSS for Windows version 25.0; IBM Corp., Armonk, NY, USA) was used for the statistical analysis. P-values less than 0.05 were considered to indicate statistical significance.

\section{RESULTS}

After open reduction, the surgical wound was treated for 2 weeks, and no side effects such as wound infection and dehiscence of the suture line were observed. The suture at the surgical site was removed at 2 weeks after surgery. Radiography performed at an average of 20 weeks (range, 18-25 weeks) after surgery revealed union of the fractured proximal phalangeal or 


\title{
Fig. 4. A case of proximal phalangeal bone fracture
}

\begin{abstract}
A 56-year-old female patient presented with a fifth proximal phalangeal bone fracture of the left hand (A). Bony alignment was maintained well in the oblique view of the radiological image. Bony union had been achieved at a postoperative follow-up of 20 weeks (B). The plate was removed at 22 weeks after open reduction surgery (C). The finger joints showed a moderate range of motion (metacarpophalangeal joint $72^{\circ}$, proximal interphalangeal joint $88^{\circ}$, distal interphalangeal joint $\left.70^{\circ}\right)(D, E)$. The operative wound had a minimal scar $(F, G)$.
\end{abstract}
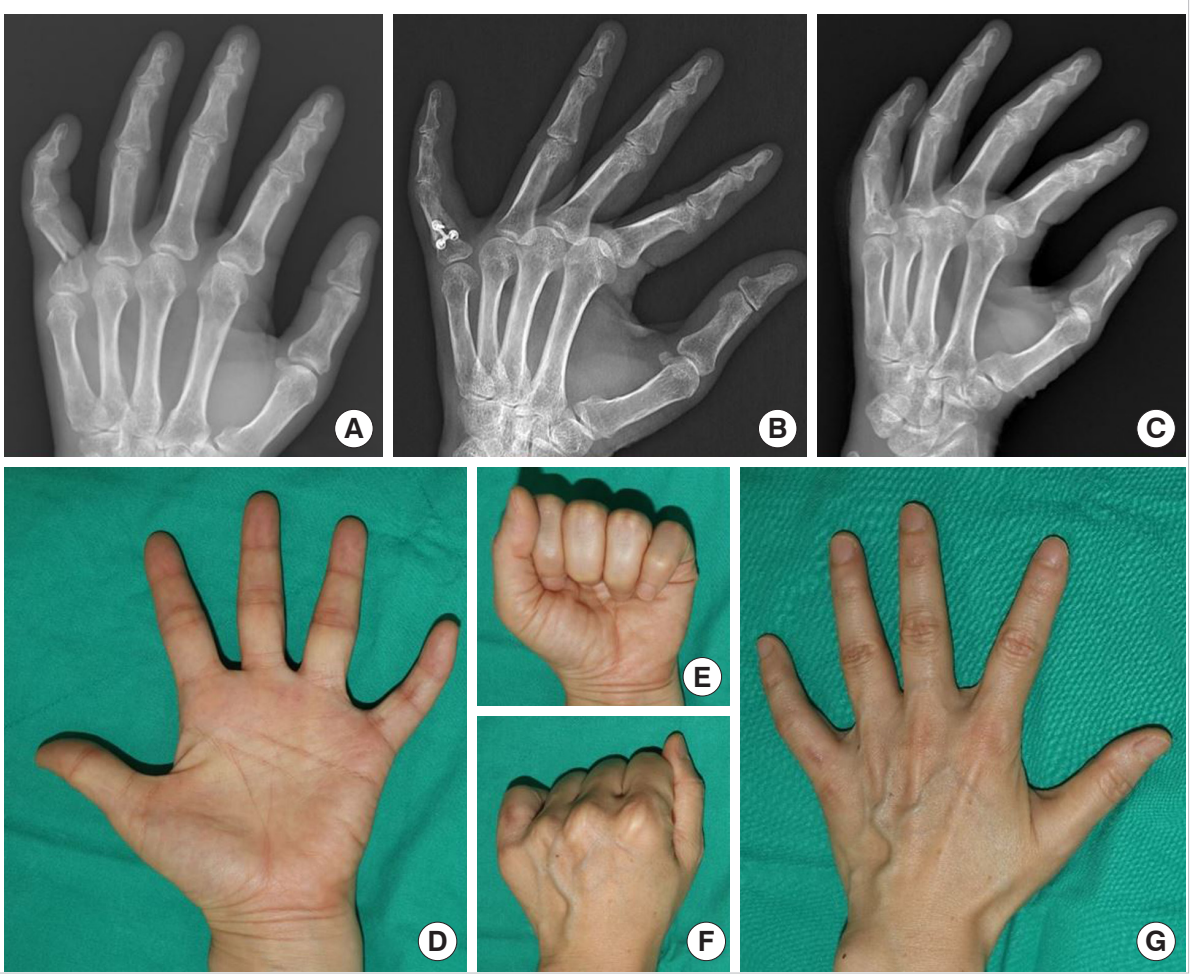

Table 2. Range of motion in proximal phalangeal bone fractures $(n=12)$

\begin{tabular}{lcccr}
\hline Measurement & ROM at 6 weeks $\left(^{\circ}\right)$ & ROM at 12 weeks $\left(^{\circ}\right)$ & P-value & Normal ROM $\left({ }^{\circ}\right)^{\text {b) }}$ \\
\hline MCP joint & $58 \pm 5(50-67)$ & $71 \pm 5(65-78)$ & $<0.001^{\text {a) }}$ & 90 \\
PIP joint & $73 \pm 6(62-81)$ & $86 \pm 3(82-88)$ & $<0.001^{\text {a) }}$ & 100 \\
DIP joint & $64 \pm 3(60-67)$ & $65 \pm 2(62-68)$ & $0.01^{\text {a) }}$ & 70 \\
Total ROM & $195 \pm 13(172-215)$ & $221 \pm 9(209-234)$ & $<0.001^{\text {a) }}$ & 260 \\
\hline
\end{tabular}

Values are presented as mean $\pm S D$ (range).

ROM, range of motion; MCP, metacarpophalangeal; PIP, proximal interphalangeal; DIP, distal interphalangeal.

${ }^{a} \mathrm{P}<0.05$; ${ }^{b}$ American Medical Association guideline, fifth edition.

metacarpal bones (Figs. 3, 4).

In the 12 patients with proximal phalangeal fractures, the range of motion improved between 6 weeks and 12 weeks after surgery for all three joints (metacarpophalangeal joint, proximal interphalangeal joint, and distal interphalangeal joint) (Table 2). The measured values were comparable to those reported in a previous study that applied two different types of exercise therapies after open reduction of proximal phalangeal fractures [10]. In that study, the sum of the range of motion of the three joints averaged $190^{\circ}$ at week 6 and $222^{\circ}$ at week 12 after surgery. In our study, the average was $195^{\circ}$ at week 6 and $221^{\circ}$ at week 12 after surgery, indicating that our exercise therapy was as effective at improving the range of motion as existing protocols for active exercise therapy.

In the 25 patients with metacarpal fractures, the range of mo- tion improved between 6 weeks and 12 weeks after surgery for all three joints (metacarpophalangeal joint, proximal interphalangeal joint, and distal interphalangeal joint) (Table 3). When compared with the results of a previous study in which exercise therapy was performed after open reduction of metacarpal fractures, the range of motion of the metacarpophalangeal joint in our study was comparable in the current study, but the sum of the range of motion of the three joints was smaller [9]. The range of metacarpophalangeal joint motion was an average of $68.5^{\circ}$ at week 6 and $82.2^{\circ}$ at week 12 after surgery in the previous study and $68^{\circ}$ at week 6 and $81^{\circ}$ at week 12 after surgery in the current study, showing a comparable level of improvement. However, the sum of the range of motion of the three joints at 12 weeks after surgery was only $236^{\circ}$ in this study, compared to the average of $256^{\circ}$ in the previous study. 
Table 3. Range of motion in metacarpal bone fractures $(n=25)$

\begin{tabular}{|c|c|c|c|c|}
\hline Measurement & ROM at 6 weeks $\left({ }^{\circ}\right)$ & ROM at 12 weeks $\left({ }^{\circ}\right)$ & P-value & Normal ROM $\left({ }^{\circ}\right)^{b)}$ \\
\hline MCP joint & $68 \pm 5(62-74)$ & $81 \pm 4(75-86)$ & $<0.001^{\text {a) }}$ & 90 \\
\hline PIP joint & $82 \pm 5(72-87)$ & $87 \pm 3(82-94)$ & $<0.001^{\text {a) }}$ & 100 \\
\hline DIP joint & $65 \pm 2(61-68)$ & $67 \pm 2(64-70)$ & $0.002^{\mathrm{a})}$ & 70 \\
\hline Total ROM & $216 \pm 10$ (195-229) & $236 \pm 8(221-250)$ & $<0.001^{\mathrm{a})}$ & 260 \\
\hline
\end{tabular}

Values are presented as mean \pm SD (range).

ROM, range of motion; MCP, metacarpophalangeal; PIP, proximal interphalangeal; DIP, distal interphalangeal.

${ }^{\text {al }} \mathrm{P}<0.05 ;{ }^{\text {b)} A m e r i c a n ~ M e d i c a l ~ A s s o c i a t i o n ~ g u i d e l i n e, ~ f i f t h ~ e d i t i o n . ~}$

Table 4. Intergroup comparison of range of motion change

\begin{tabular}{lccc}
\hline & \multicolumn{2}{c}{ ROM change $\left(^{\circ}\right)$} & \\
\cline { 2 - 3 } Measurement & $\begin{array}{c}\text { Proximal } \\
\text { phalangeal bone } \\
\text { fracture group }\end{array}$ & $\begin{array}{c}\text { Metacarpal bone } \\
\text { fracture group }\end{array}$ & P-value \\
\hline MCP joint & $13 \pm 2(11-16)$ & $13 \pm 3(8-16)$ & 0.7 \\
PIP joint & $13 \pm 4(7-20)$ & $5 \pm 3(2-14)$ & $<0.001^{\text {a) }}$ \\
DIP joint & $2 \pm 1(0-3)$ & $2 \pm 1(0-4)$ & 0.43 \\
Total ROM & $27 \pm 5(19-37)$ & $20 \pm 5(9-33)$ & $<0.001^{\text {a) }}$ \\
\hline
\end{tabular}

Values are presented as mean \pm SD (range).

ROM, range of motion; MCP, metacarpophalangeal; PIP, proximal interphalangeal; DIP, distal interphalangeal.

a) $P<0.05$.

The sum of the range of motion of the three joints (metacarpophalangeal joint and proximal and distal interphalangeal joints) at 12 weeks exceeded $230^{\circ}$ in 26 patients (five cases in the proximal phalangeal fracture group and 21 cases in the metacarpal fracture group).

An intergroup comparison between the proximal phalangeal bone fracture and the metacarpophalangeal bone fracture groups was performed using data on the change in range of motion in each joint. The two groups did not show statistically significant differences for the metacarpophalangeal and distal interphalangeal joints (Table 4). The proximal interphalangeal joint was altered with a greater range in the proximal phalangeal bone fracture group than in the metacarpal bone fracture group $\left(13^{\circ} \pm 4^{\circ}\right.$ vs. $\left.5^{\circ} \pm 3^{\circ}, \mathrm{P}<0.05\right)$. The range of motion at 6 weeks was smaller in the proximal phalangeal bone fracture group than in its counterpart $\left(73^{\circ} \pm 6^{\circ}\right.$ vs. $\left.82^{\circ} \pm 5^{\circ}, \mathrm{P}<0.05\right)$. Nonetheless, the range of motion at 12 weeks was comparable $\left(86^{\circ} \pm 3^{\circ}\right.$ vs. $\left.87^{\circ} \pm 3^{\circ}, \mathrm{P}>0.05\right)$.

\section{DISCUSSION}

The surgical approach, as well as the reduction and fixation method, is an important factor to consider in the surgical treatment of hand fractures, as the extent of skin and soft tissue incision and periosteal and peritendinous dissection can be affected by the surgical approach $[4,11]$. According to the literature on surgical approaches, the internal plate fixation method seems to exhibit superior strength to the external pin fixation method [12]. However, as open reduction and internal fixation involves a skin incision and peritendinous dissection, care should be taken to minimize tissue damage during the incision and dissection, and some surgeons prefer less invasive surgical methods for this reason.

Due to the differences among previous studies in terms of the fracture pattern and the extent of incision and dissection for open reduction, no single approach can be considered optimal [13]. Therefore, it is advised to choose an appropriate technique for each case, rather than only considering either closed reduction and external fixation or open reduction and internal fixation. Further research is necessary, especially on minimally invasive methods of open reduction.

For internal fixation after open reduction, using a thin plate enables proper suturing of the periosteum, which facilitates tendon gliding and early postoperative rehabilitation [14]. If a thick plate is used on the phalangeal or metacarpal bones for firm fixation, the plate may restrict tendon gliding or joint motion and cause pain. Moreover, in a biomechanical study that compared the miniplates and microplates used in phalangeal and metacarpal fractures, microplating showed comparable fixation strength under stress [15].

Furthermore, it is important to carry out appropriate exercise therapy at a suitable time, since stability changes during the postoperative recovery period. According to the postoperative rehabilitation guidelines in the existing literature, moderate pain-free functional activities with a fracture brace and controlled protected reactivation therapy are recommended at 3 weeks after surgery, which is when fibrocartilage tissue forms. At 3-6 weeks after surgery, when the peripheral bones form, active protected reactivation is possible [13].

Exercise therapies feasible at 2-3 weeks after surgical treatment of metacarpal fractures include massaging the affected area, warm bathing of the hand (exercises performed with the hand placed in warm water), grasping the unaffected hand with 
the affected hand, pinching with the thumb and the affected finger, and bending and stretching the fingers with the entire arm stretched above the head [9]. In addition to these conventional exercises, we hypothesized that a traction exercise in which a load is applied against the direction of axial loading on the fractured proximal phalangeal or metacarpal bone would be possible at 3 weeks after surgery. The metacarpophalangeal and interphalangeal joints were flexed so that the distal phalanges and the palmar aspect of the metacarpal bones could form a right angle, and an elastic band was placed on the distal and middle phalanges for the traction exercise (Fig. 1). Of the intrinsic muscles of the hand, the lumbricals arise from the flexor digitorum profundus and insert into the extensor hood; thus, even without strong flexion of the phalanges, shoulder and elbow joint exercises can be performed against the traction of the elastic band hung around the distal and middle phalanges [16].

Isometric exercises have been utilized to treat joint tendinopathy, which causes limited motion. Stasinopoulos and Stasinopoulos [17] performed isometric contractions of the wrist joint, combined with eccentric-concentric exercise in lateral elbow tendinopathy patients [17]. The exercise protocol resulted in functional improvement and reduced pain. Our exercise using the elastic band is based on common knowledge, since the affected joint is in the isometric state, while the adjacent elbow and shoulder joints undergo eccentric and concentric movements. Nagano et al. [18] noted that isometric exercises increased the range of motion of ankle joints in healthy men and women. They considered that inhibition of the Golgi tendon organ or stretch tolerance led to these effects of isometric exercise.

The improvement of the postoperative range of motion in proximal phalangeal fractures in this study was comparable to that reported in a previous study. However, in the metacarpal fractures, while the range of motion of the metacarpophalangeal joints was similar to that reported in a previous study, the entire range of motion of the three joints was smaller in the current study. This may be attributed to the fact that we focused on the metacarpophalangeal joint and the balanced action of the intrinsic and extrinsic muscles of the hand in exercise therapy, while neglecting the range of flexion of the proximal and distal interphalangeal joints.

In the intergroup analysis, the proximal interphalangeal joint in the proximal phalangeal bone fracture group showed greater change than in the metacarpophalangeal bone fracture group. The range of motion at 6 weeks was more restricted in the proximal phalangeal bone fracture group; nonetheless, regular exercise therapy led to comparable outcomes in both groups. The limited range of motion in the early phase can result in finger stiffness, and appropriate exercise following operative treatment should be considered for each joint [19].

After performing an active traction exercise involving three positions of the hand (supination, neutral, and pronation) as described above, the patients were instructed to exercise on their own using an elastic band after returning home. This decision was based on a previous report, which suggested that both traditional physical therapy and a home exercise program were effective in postoperative management [9]. We conjectured that since rotation vectors are present in the phalangeal and metacarpal bones, various extrinsic and intrinsic muscles of the digits would produce complex interactions [20]. Thus, we intended to stimulate each muscle through exercises involving three different positions of the hand (Fig. 1). Furthermore, moderate functional activities were allowed starting 4 weeks after surgery.

At approximately 3 weeks after surgery, when the fibrocartilage starts to form, appropriate exercise therapy should be applied according to the condition of the fracture, age, underlying conditions, and motor function; moreover, the direction of displacement according to the fracture type is also an important consideration $[11,20]$. According to the literature on the direction of angulation in hand fractures, metacarpal fractures tend to have an apex dorsal angulation, whereas proximal phalangeal fractures tend to have an apex volar angulation [21]. The direction of angulation is affected by the function of the associated tendons. Among the intrinsic muscles of the hand, the interosseous muscle, which arises from the metacarpal bone and inserts into the extensor hood, is known to play a particularly important role in post-fracture displacement. If a patient complains of pain during exercise therapy or there is a possibility of worsening displacement depending on the fracture type, other exercise therapies should be considered. We also took into account the direction of displacement and, depending on the type of fracture, added an exercise in which a thermoplastic splint was applied to the proximal phalanx and active extension and passive flexion exercises were performed using the tension of the rubber band (Fig. 2). Extension contracture of the metacarpophalangeal joint is a problematic phenomenon that follows hand injuries, and the intrinsic-plus position is advocated to focus on functional balance [22]. The light tension of the rubber band led to passive flexion of the metacarpophalangeal joint, and patients in the recovery phase could generate sufficient power to overcome the resistance.

The main limitation of this study is the lack of a comparison with other groups that underwent different surgical methods or exercise therapies for proximal phalangeal or metacarpal fractures. Although the range of motion of the interphalangeal and metacarpophalangeal joints was analyzed over time during the 
postoperative period, it is necessary to identify appropriate exercise therapies conducive to improving the range of motion of each joint through an objective comparison with other treatment methods. In this context, a comparative analysis using closed reduction and the external pinning technique, as well as alternative exercises, would be promising areas for further research.

Another limitation is that we performed measurements simultaneously with regard to two different exercise protocols, which focused on different anatomic structures. Nonetheless, the exercises are based on a common rationale that minimized loading on intrinsic muscles, and utilized them as a source of stability [16]. The intrinsic muscles can work similarly to the suspension wire of cable bridges. Furthermore, previous studies have presented post-exercise analyses after combined exercise protocols $[9,23]$.

Before peripheral bone formation after surgery, hand exercises that place the least possible load on the fracture site are recommended. Based on our findings, pain-free traction exercises and extension and flexion exercises of the metacarpophalangeal joint, as well as conventional exercise therapies, will contribute to the rehabilitation of patients with hand fractures.

Minimal periosteal and peritendinous dissection is an advantage of minimally invasive open reduction and internal fixation for proximal phalangeal or metacarpal fractures. When limited stability is achieved, at 3 weeks after internal fixation, appropriate active exercises that do not cause dislocation of the fracture site should be performed. Active traction exercises, which apply a load against the direction of axial loading, along with active extension and passive flexion exercises of the metacarpophalangeal joint, facilitated early active reactivation. Compared to previously described exercise therapies, the controlled active exercise described herein resulted in comparable improvements in the range of motion. Further research on various hand exercises is needed.

\section{NOTES}

\section{Conflict of interest}

No potential conflict of interest relevant to this article was reported.

\section{Ethical approval}

The study was approved by the Institutional Review Board of Konkuk University Medical Center (IRB No. KUMC 2020-03013) and performed in accordance with the principles of the Declaration of Helsinki. Written informed consent was obtained.

\section{Patient consent}

The patients provided written informed consent for the publication and the use of their images.

\section{Author contribution}

Conceptualization: H Choi, M Lee. Data curation: D Jun. Formal analysis: J Bae, D Shin, H Choi, J Kim. Methodology: J Bae, D Shin, H Choi, J Kim. Visualization: J Kim. Writing - original draft: D Jun, J Bae, M Lee. Writing - review \& editing: D Shin, H Choi, J Kim.

\section{ORCID}

Dongkeun Jun https://orcid.org/0000-0001-9017-9929

Jaehyun Bae https://orcid.org/0000-0001-8716-3109

Donghyeok Shin https://orcid.org/0000-0002-8450-4411

Hyungon Choi https://orcid.org/0000-0002-3816-1286

Jeenam Kim https://orcid.org/0000-0002-4080-6135

Myungchul Lee https://orcid.org/0000-0002-9721-0092

\section{REFERENCES}

1. Kim JK, Kim DJ. Antegrade intramedullary pinning versus retrograde intramedullary pinning for displaced fifth metacarpal neck fractures. Clin Orthop Relat Res 2015;473: 1747-54.

2. Reformat DD, Nores GG, Lam G, et al. Outcome analysis of metacarpal and phalangeal fixation techniques at Bellevue Hospital. Ann Plast Surg 2018;81:407-10.

3. Strauch RJ, Rosenwasser MP, Lunt JG. Metacarpal shaft fractures: the effect of shortening on the extensor tendon mechanism. J Hand Surg Am 1998;23:519-23.

4. Logters TT, Lee HH, Gehrmann S, et al. Proximal phalanx fracture management. Hand (NY) 2018;13:376-83.

5. Beutel BG, Ayalon O, Kennedy OD, et al. Crossed K-wires versus intramedullary headless screw fixation of unstable metacarpal neck fractures: a biomechanical study. Iowa Orthop J 2018;38:153-7.

6. Bao B, Zhu H, Zheng X. Plate versus Kirschner wire fixation in treatment of fourth and fifth carpometacarpal fracturedislocations: a retrospective cohort study. Int J Surg 2018; 52:293-6.

7. Biz C, Iacobellis C. Comparison of percutaneous intramedullary Kirschner wire and interfragmentary screw fixation of displaced extra-articular metacarpal fractures. Acta Biomed 2014;85:252-64.

8. Kose A, Topal M, Engin MC, et al. Comparison of low-profile plate-screw and Kirschner-wire osteosynthesis outcomes in extra-articular unstable proximal phalangeal fractures. Eur 
J Orthop Surg Traumatol 2019;29:597-604.

9. Gulke J, Leopold B, Grozinger D, et al. Postoperative treatment of metacarpal fractures: classical physical therapy compared with a home exercise program. J Hand Ther 2018;31:20-8.

10. Miller L, Crosbie J, Wajon A, et al. No difference between two types of exercise after proximal phalangeal fracture fixation: a randomised trial. J Physiother 2016;62:12-9.

11. Freeland AE, Orbay JL. Extraarticular hand fractures in adults: a review of new developments. Clin Orthop Relat Res 2006;445:133-45.

12. Chiu YC, Tsai MT, Hsu CE, et al. New fixation approach for transverse metacarpal neck fracture: a biomechanical study. J Orthop Surg Res 2018;13:183.

13. Skirven TM, Osterman AL, Fedorczyk JM, et al. Rehabilitation of the hand and upper extremity. 6th ed. Philadelphia: Elsevier Health Sciences; 2011.

14. Puckett CL, Welsh CF, Croll GH, et al. Application of maxillofacial miniplating and microplating systems to the hand. Plast Reconstr Surg 1993;92:699-707.

15. Prevel CD, Eppley BL, Jackson JR, et al. Mini and micro plating of phalangeal and metacarpal fractures: a biomechanical study. J Hand Surg Am 1995;20:44-9.

16. Arnet U, Muzykewicz DA, Friden J, et al. Intrinsic hand muscle function, part 1: creating a functional grasp. J Hand Surg Am 2013;38:2093-9.

17. Stasinopoulos D, Stasinopoulos I. Comparison of effects of eccentric training, eccentric-concentric training, and eccentric-concentric training combined with isometric contraction in the treatment of lateral elbow tendinopathy. J Hand Ther 2017;30:13-9.

18. Nagano K, Uoya S, Nagano Y. Effects of antagonistic muscle contraction exercises on ankle joint range of motion. J Phys Ther Sci 2019;31:526-9.

19. Lutsky KF, Matzon JL, Dwyer J, et al. Results of operative intervention for finger stiffness after fractures of the hand. Hand (N Y) 2016;11:341-6.

20. Barry AJ, Murray WM, Kamper DG. Development of a dynamic index finger and thumb model to study impairment.J Biomech 2018;77:206-10.

21. Chang J, Neligan PC, Warren RJ, et al. Plastic surgery. Philadelphia: Elsevier Saunders; 2012.

22. Rongieres M. Management of posttraumatic finger contractures in adults. Hand Surg Rehabil 2018;37:275-80.

23. Gutierrez-Espinoza H, Rubio-Oyarzun D, Olguin-Huerta C, et al. Supervised physical therapy vs home exercise program for patients with distal radius fracture: a single-blind randomized clinical study. J Hand Ther 2017;30:242-52. 